\title{
Ageing bone fractures: the case of a ductile to brittle transition that shifts with age
}

\author{
Peter Zioupos ${ }^{1 *}$, Helmut O.K. Kirchner2 ${ }^{2}$ Herwig Peterlik ${ }^{3}$ \\ ${ }^{1}$ Cranfield Forensic Institute, Cranfield University, DA of the UK, Cranfield University, Shrivenham, UK \\ 2 INM- Leibniz Institute for New Materials, Campus D22, D-66123 Saarbruecken, Germany \\ ${ }^{3}$ Faculty of Physics, University of Vienna, Vienna, Austria
}

\begin{abstract}
Human bone becomes increasingly brittle with ageing. Bones also fracture differently under slow and fast loadings, being ductile and brittle, respectively. The effects of a combination of these two factors have never been examined before. Here we show that cortical bone is most fracture-resistant at the physiologically prevalent intermediate strain rates of $10^{-3} \mathrm{~s}^{-1}$ to $10^{-2} \mathrm{~s}^{-1}$ such as they occur in walking or running, slightly weaker at slower quasistatic and much weaker at fast impact loading rates. In young cortical bone (15 years of age) the ductile-to-brittle transition (DBT) occurs at strain rates of $10^{-2} \mathrm{~s}^{-1}$, in old cortical bone (85 yrs) at speeds lower by a factor of 10 to 40 . Other research has shown that the energy required to break bone (per unit of fracture surface) drops as much as $60 \%$ between these two ages. Therefore, DBT seems to compound the well-known phenomenon of 'brittle old bones'. Old bones can only cope with slow movement, young ones with both slow and fast movement. These observed material characteristics of (i) a shift of the DBT and (ii) a reduced energy absorption capacity appear to contribute at least as much to the loss of bone quality as the various quantity based (lowered bone density and mineral content) explanations of the past. They also provide a new powerful paradigm, which allows us to demonstrate mechanically, and uniquely, how human bone becomes increasingly brittle with age.
\end{abstract}

Keywords: $\quad$ bone; ageing; toughness; strain rate, fractures; biomechanics; evolution;

* Corresponding author at: Cranfield Forensic Institute, Cranfield University, Defence Academy of the UK, Shrivenham SN6 $8 L A$ UK E-mail address: p.zioupos@cranfield.ac.uk (P.Zioupos)

\section{Introduction}

The frequency of fractures (hip, spine, and wrist) increases dramatically with age [1]. This is an acute public health problem and will be even more so because of the increasing life expectancy of the population resulting, for instance, in an increase in hip fractures by a factor of seven by 2050 [2]. The increased vulnerability could be due to a number of factors some of which, like the deteriorating reflexes of the elderly and the reduced proprioceptive ability leading perhaps to more frequent falls, spring to mind easily (Fig.1). However, when the incidents are normalised for the number of accidental falls the elderly show that they break bones more frequently than the young and this then clearly is due to some kind of deterioration in bone quality $[3,4]$ too. However, what should be taken as a measure of bone quality is not immediately clear. Bone conditions like osteoporosis (OP), define the problem in terms of reduced bone density, but this only explains about $70-80 \%$ of the variability in the fracture data $[1,3]$. Changes in the mineral phase of OP bone [4] and remedial action to affect its remodelling cycles [5] go some way towards describing the cause of the problem but do not explain what is at the root of it and how best to quantify 'quality' in mechanical performance.

People who show no specific bone disease show a dramatic $60 \%$ decrease in the energy absorbing capacity of bone tissue in the years following skeletal maturity [6], but the problem is that the vast majority of such data in literature of bone property changes refers to tests carried out in quasistatic loading $[3,6]$ or in impact [6,7], but rarely alongside each other or over a wider strain range. In life bone continuously remodels itself and keeps on changing as material. It also breaks either in impact or following the fast propagation of crack initiated by fatigue. Meanwhile in different parts of the skeleton bones experience a wide range of strain rates. On one end of the spectrum, quasistatic rates of 
about $10^{-4} \mathrm{~s}^{-1}$ occur when we carry shopping bags, turn over in sleep, squat, or raise ourselves from a sitting position. At the other end, much faster rates up to $10 \mathrm{~s}^{-1}$ and beyond, are experienced in falls, or in accidental shock impact like in car accidents, which have attracted attention in recent biomechanics research $[8,9]$. Between the two strain rate extremes on a daily basis, we expose our bones to physiological strain rates between $10^{-3}$ and $10^{-2} \mathrm{~s}^{-1}$ as in the tibia when we walk or jump [10]; in the skull and the mandible when we masticate [11]. So far, all biomechanical experiments on bone have been performed only at low or fast extremes and rarely throughout the full range with emphasis on the physiologically relevant rates. The energy to fracture has been shown to decrease from $4 \mathrm{~kJ} \mathrm{~m}^{-2}$ by $60 \%$ between $35-92$ yrs of age at quasistatic loading; while in impact rates decreased from $2 \mathrm{~kJ} \mathrm{~m}^{-2}$ down by $10 \%$ in notched specimens and from $12 \mathrm{~kJ} \mathrm{~m}^{-2}$ down by $33 \%$ in un-notched specimens, over the same age range $[6,12]$. These values are of relatively little value for the management of fractures as few people break a bone during sleep or carrying a shopping bag (creep rupture conditions) and almost everyone fractures one in a car crash [13]. What happens in the intermediate rates is what matters. Strain rate is not only of medical, but also of physical importance. Animal bone, be it bovine femoral [14], porcine mandibular [15], bovine tibial [16] or equine metacarpal [17] shows strong strain rates effects like many industrial materials [18]. At low rates fracture is ductile and the energy under the stress/strain curve increase with strain rate; as strain rate increases further fracture becomes brittle and the energy absorbed reduces $[17,19]$. Strong indications that bone has reduced toughness at higher strain rates have appeared in some computational and experimental studies $[20,21,22,23]$.

We have conducted in the past tests on specimens of human femoral cortical bone at a single age loaded in tension [19] at strain rates ranging from low $\left(0.08 \mathrm{~s}^{-1}\right)$ to high $\left(18 \mathrm{~s}^{-1}\right)$. Across this strain rate range the modulus of elasticity generally increased, stress at yield and failure and strain at failure decreased for rates higher than $1 \mathrm{~s}^{-1}$, while strain at yield was invariant for most strain rates and only decreased at rates higher than $10 \mathrm{~s}^{-1}$. The results showed that strain rate has a strong effect on post-yield deformation, i.e. the capacity or bone for plasticity and ductility. Specimens loaded at high strain rates were brittle, while those loaded at low strain rates were much tougher. Post-test examination of the microcracking damage that ensued during the tests revealed that the amount of microcracking was inversely related to the strain rate [24]. Specimens loaded at low strain rates showed considerable postyield strain and also much more microcracking. Partial correlation and regression analysis suggested that the development of post-yield strain was a function of the amount of microcracking incurred (the cause), rather than being a direct result of the strain rate (the excitation); the reason being that low strain rates allowed time for microcracking to develop, which increased the compliance of the specimens, making them tougher. These results showed that the degree to which bone is brittle or tough depends on the amount of microcracking damage it is able to sustain, or in other words its ability to avoid a ductile-to-brittle transition for as long as possible during the deformation. The combination of microcracking damage studies and toughness measurements showed that the key to bone's brittleness is whether or not there is a strain- and damage-localisation early on in the loading process, which leads to low post-yield strains and low-energy absorption to failure.

The combination of observing the ensuing microcracking damage, its localisation and the concomitant end result (brittleness) makes then the basic reason for this ductile-to-brittle transition (DBT) physically clearer. At small speeds of deformation plasticity, which is rate-dependent, either because it requires mass transport or because of viscoelastic dissipation of peak stresses, is fast enough to screen the elastic field around a crack and the 'plastic' zone toughens the material. At high loading rates plasticity cannot keep up and the elastic fields prevail, leading to brittle fracture. Bone with its hierarchical structure [25] allows for multiple toughening mechanisms either before or after the creation of a major crack [26] by means of viscoelastic flow, microcracking, crack bridging and crack deflection [27]. In human femoral bone, so far only the decrease of strength with strain rate at medium to high stain rates has been reported, but this has not been described as a function of the age of the individual [19] and the increase of toughness with the strain rate at the slow side has not been ascertained. Human data on a surmised DBT behaviour is still lacking and animal studies, which dominate the biomechanics field, are not appropriate for drawing conclusion on age effects that may affect human tissue.

It appears that the combined effects of ageing and strain rate have never been examined before although they promise to be particularly relevant for medical and epidemiological purposes. Arguably, bone in-vivo is mechanically subjected to bending, tension, and compression and might respond differently to these loadings in terms of stiffness and strength. However, the most valid and probably the most clinically relevant parameter to express its resistance to fracture is the energy absorption (what we might call 'toughness') prior to failure. This is what needs to be quantified as a function of both age and strain rate (rate of loading) and 
such experiments have never been reported in the literature. Our aim, therefore, is threefold: first to explore whether there is a DBT in human bone and observe the likely transition point with respect to physiological in-vivo loading rates such as these in our everyday life; second, to verify a shift to lower rates with respect to age, as we suspect that old bones might somehow be more brittle than young ones; third, to examine how absolute values of fracture resistance vary with age and strain rate.
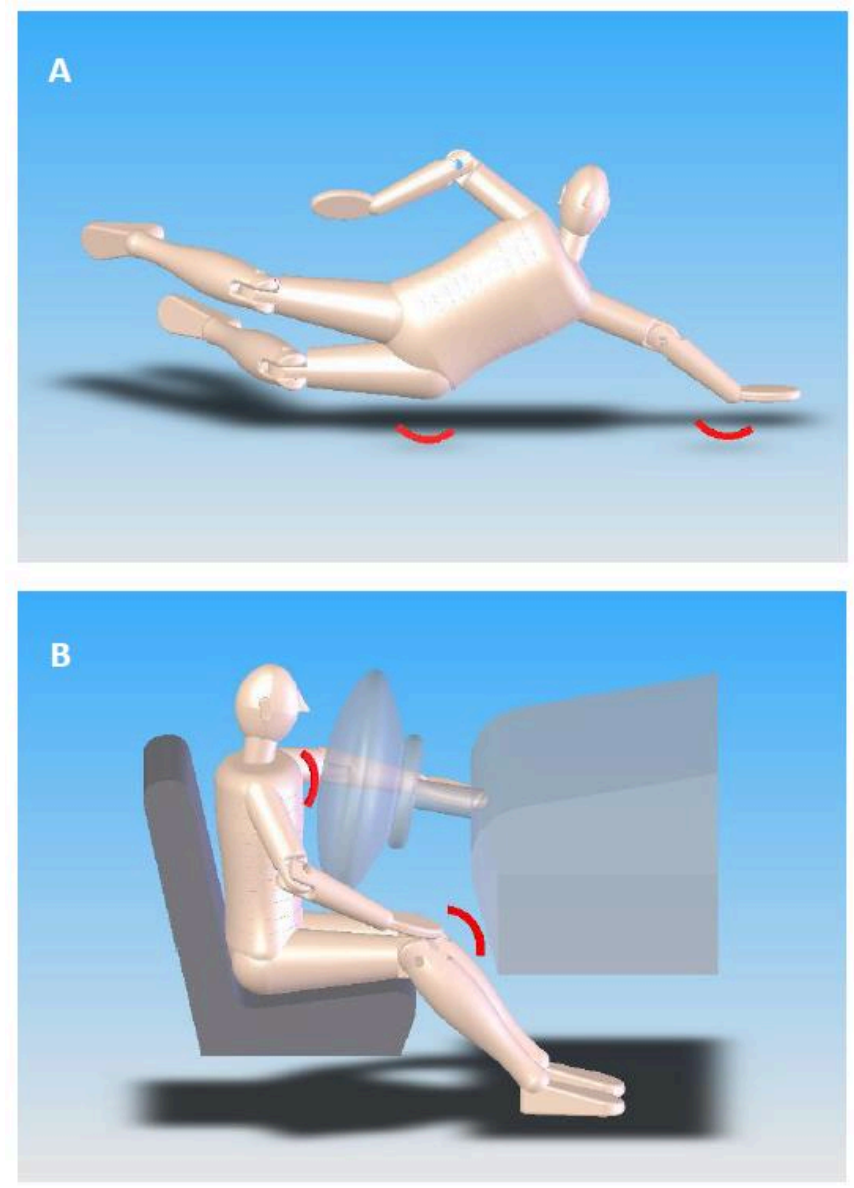

Figure 1. Epidemiology shows that the frequency of fractures increases dramatically with age. Accidental traumatic fractures of the nature depicted here occur often (A) through slips and falls (neck of femur fracture, or Colle's fractures or the wrist as one stretches out to protect against impact with the ground) or even (B) due to events in our modern motorised lifestyle and environment. In evolutionary terms, and to a certain extent, the skeleton is perhaps expected to cope with events like (A), but it is not meant to cope with very fast events (taking place in less than $10 \mathrm{~ms}$ ) like in a car crash (B). (Illustrations courtesy of Dr E Minnock.)

\section{Materials \& methods}

The samples were from donors of selected ages at the start of skeletal maturity (15) and then after skeletal maturity $(55,64,75,85)$ when properties gradually decline. The donors had died of conditions unrelated to the condition of the skeleton. They had been donated under informed consent to a tissue bank and with the relevant guidelines and regulations being followed as stipulated by legal procedures. Ethical permission was obtained by the Gloucester and Cheltenham NHS Trust hospitals committee and then in further protocols by Frenchay Bristol for the BOSCOS (Bone Scanning for Occupant Safety - Mr.C.Curwen CI REC ref. 01/179G) project supported by the UK Department of Transport; and later by the by EPSRC (UK) for 'Point-of-Care High Accuracy Fracture Risk Prediction' programme. The samples were prepared in the form of beams $6 \mathrm{~mm}$ wide and $3 \mathrm{~mm}$ deep and $40 \mathrm{~mm}$ long, dissected from the mid diaphysis and orientated along the axis parallel to the axis of the femur (Fig. 2). This method devised by Tattersal \& Tappin [28] to determine the work of fracture of brittle materials and has been used quite often on mineralised tissues [29,30,31,32,33]. This method is similar to various fracture mechanics configurations in that, among other things, it aims to restrict damage in the specimen to a very small part of it, so that what is being measured is the work associated with the travelling fracture, and not damage in more remote parts of the specimen.

The samples contained a chevron notch in the middle of the span and they were bent at various speeds in a servo hydraulic material testing machine. The crosshead speeds can be converted to strain rate by simple beam theory. The work to fracture $\left(W_{f}\right)$ is obtained by dividing the area under the load-displacement curve by the ligament area of intact tissue to derive an energy density measure $\left(\mathrm{kJ} \mathrm{mm}^{-2}\right)$ necessary for breaking the bone, $W_{f}$. It measures the energy required to create a unit of fracture surface and thus the toughness of the tissue in the sense that it defines what it takes to sustain a stable crack growth $[34,35]$. This is to distinguish it from other uses of the term toughness in classical fracture mechanics methods, which employ $\mathrm{K}_{\mathrm{C}}$ the critical stress intensity factor, and/or the J-integral and define conditions that are necessary to be present at the start of the crack growth process.

The loading rate in the three-point bending test of a beam of span $L$, breadth $b$ and thickness $h$, is converted to strain rate as for beam theory [36], where the load $F$ in the middle of the beam of length $L$ produces a moment:

$\mathrm{M}=\mathrm{F} \mathrm{L} / 4$

The maximum displacement is at the central point:

$\delta=\mathrm{F} \mathrm{L}^{3} / 48 \mathrm{E} \mathrm{I}$,

where $E$ is the modulus of elasticity and $I\left(=\mathrm{b} \mathrm{h}^{3} / 12\right)$ the moment of inertia of the beam. 

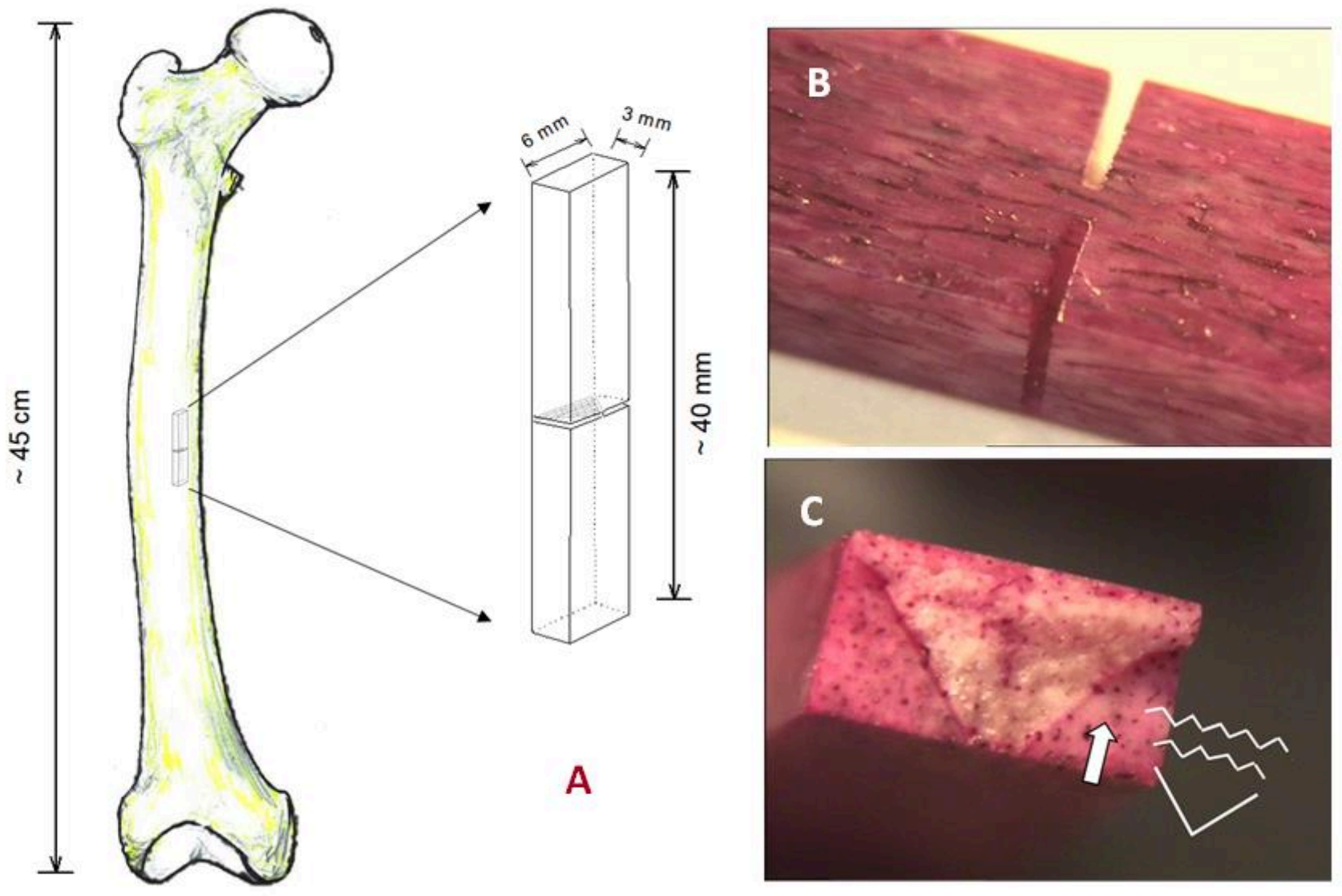

Figure 2. (A) The samples were shaped in the form of beams 6 $\mathrm{mm}$ wide and $3 \mathrm{~mm}$ deep and $40 \mathrm{~mm}$ long, dissected from the mid diaphysis and orientated along the axis of the femur. A chevron notch was prepared in the middle of the span and they were fractured in bending at various speeds in material testing machine. This specimen design and geometry drives a crack along a transverse plane, perpendicular to the long axis of the bone. (B) The chevron notch was prepared in a V-shape in the middle of the span with a metallurgical low speed saw. (B) before and (C) after the fracture test. This sample has been stained with Fuchsin (a histological stain) to demonstrate the vasculature (in dark red) of normal femoral bone material. The V-shaped notch helps to stabilise the rate of crack growth because the advancing crack front (in the direction of the white arrow) meets an ever-increasing width of material ahead of it.

The longitudinal stress varies linearly across the beam and reaches a maximum at the surface,

$$
\sigma=\mathrm{M} \mathrm{h} / 2 \mathrm{I}=\mathrm{FLh} / 8 \mathrm{I}=12 \mathrm{~F} \mathrm{~L} \mathrm{~h} / 8 \mathrm{~b} \mathrm{~h}^{3}=(3 / 2) \mathrm{F} \mathrm{L}
$$
$/ \mathrm{b} \mathrm{h}^{2}$

The strain is :

$\varepsilon=\sigma / \mathrm{E}=\mathrm{Mh} / 2 \mathrm{El}=\mathrm{FLh} / 8 \mathrm{El}=\left(\mathrm{FL}^{3} / 48 \mathrm{El}\right) 6$ $\mathrm{h} / \mathrm{L}^{2}=\delta 6 \mathrm{~h} / \mathrm{L}^{2}$
The relationship between displacement and the maximum strain is therefore:

$\varepsilon=\delta 6 \mathrm{~h} / \mathrm{L}^{2}$

For a span of $L=32 \mathrm{~mm}$ and a depth of the beam of $h=3$ $\mathrm{mm}$ the ratio between displacement and strain and therefore between stroke rate and strain rate is therefore $\sim 57: 1$.

Because of the chevron V-shaped notch the Tattersal \& Tappin test [28] has been thought as being ideal for driving stable cracks through a material. This is practically true but not strictly so, because of two added factors: (i) using a beam design means that as the crack advances through the beam the strain rate changes slightly because the loading geometry changes due to reduction in effective thickness, therefore there are deviations from the ideal formulae mentioned above and even if one exerts a constant stroke rate $(\mathrm{d}$ ] $/ \mathrm{dt})$ one is not guaranteed to produce constant strain rate (d目/dt); (ii) due to the finite compliance of the grips of the testing machine, as the effective thickness reduces the compliance of the sample increases and ratio to the machine compliance will proportionally decrease putting slightly more pressure on the sample and causing the crack to accelerate somewhat. 


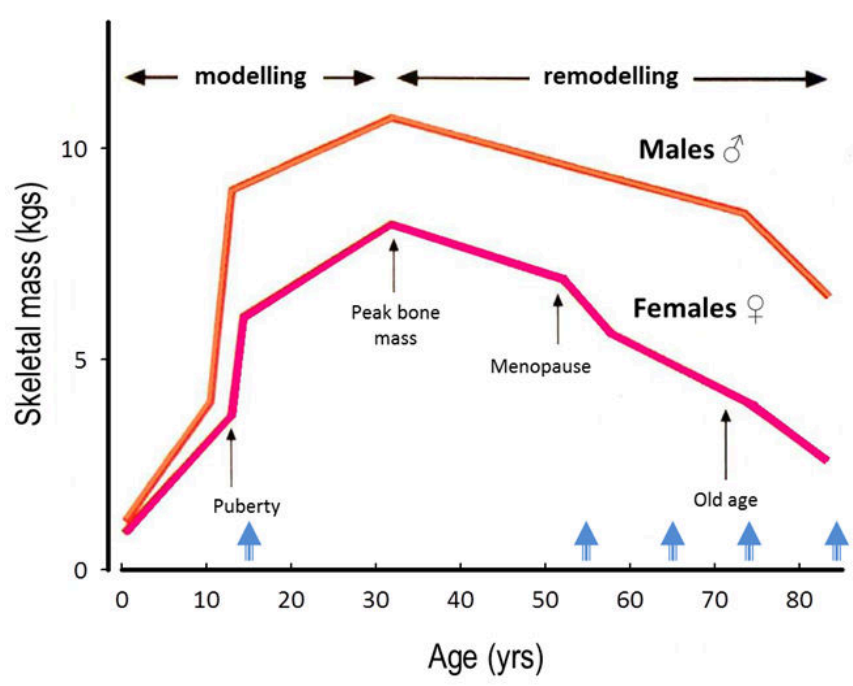

Figure 3. The overall mass of the skeleton shows certain trends with age. Bones are at their peak mass between 15 and 50 yrs of age declining in mass either side of this interval. The underlying concomitant changes in bone quality are also significant but much less obvious [4]. One must keep in mind that modelling and remodelling apply throughout life and the shown ranges should more accurately referred to as perhaps 'predominantly' modelling and remodelling activity ages. Samples were taken from donors at 15yrs of age (at the start of skeletal maturity) and then from donors at intervals nearly every 10 years after maturity, at 55, 64, 75, 85 yrs. This graph was compiled by well-known trends in the literature and the weight axis was adjusted for the body weight of the subjects of this study.

\section{Results \& discussion}

3.1 Mechanical tests We successfully tested altogether 113 specimens cut from five male human femurs by the Tattersal \& Tappin test method to produce work of fracture toughness values $\left(W_{f}\right)$. Other associated material and chemical information on the samples was also collected to help in assessing the ontogenic changes and put the results into context (esupplementary information). The samples were from donors of ages between 15 (start of skeletal maturity) and at 55, 64, 75, 85 yrs (every 10 yrs after skeletal maturity when properties gradually decline) (Fig. 3). The donors had died of conditions unrelated to the condition of the skeleton and the result is therefore, representative of normal healthy femurs at various ages. The age effects on the bone material quality show more clearly in males, because in females the inter-individual variability caused by the varying number of pregnancies, breast feeding, menopause etc. do not allow for the selection of a representative bone specimen for material analysis at any particular age. It is also true that although most OP fracture sites involve mostly cancellous bone, the study of bone properties at the basic material science and physics level come inevitably from studying cortical bone. Cortical bone provides the material bone mass from which all bone is made, cancellous and cortical, without the convoluted effects of wide porosity variations and broad architectural changes seen in cancellous bone.

\subsection{Strain rate and timescale of events}

Implementing a range of strain rates in the lab requires a meaningful translation of displacements rates to strain rates and the timescale of events so that the tests are easy to interpret. The rate of conversion between displacement $\delta$, and strain $\varepsilon$, in our test configuration was $(\mathrm{d}$ ? $/ \mathrm{dt}):(\mathrm{d}$ ? $/ \mathrm{dt}) \sim 57: 1$. The loading rates were representative of actual intentionally or accidentally applied loadings of the femur, and so were the times of fracture (the time it took to break any single specimen was analogous to completely fracturing a whole bone in a similar situation in-vivo). This discussion is pertinent as the relative magnitude of strains, deformations and the time scale of events (Fig. 4) is critical for setting the present test results in the right context.

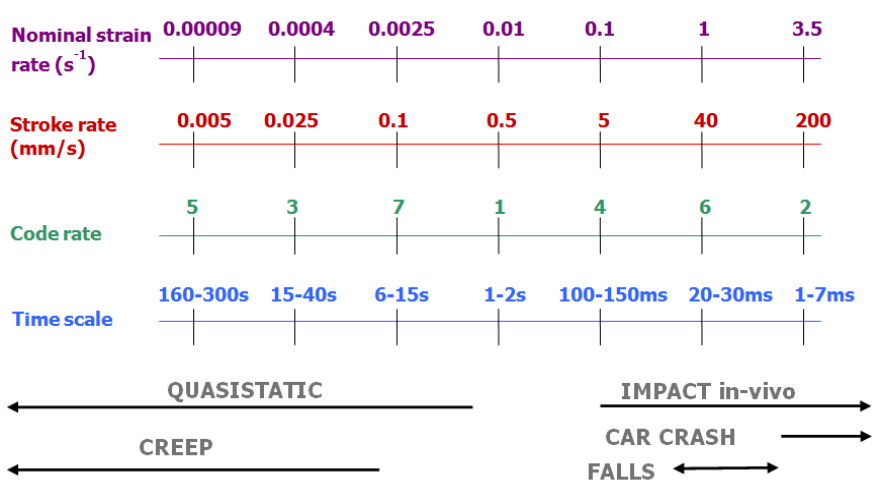

Figure 4. Considerations for the relative scale for strain rates, stroke (deformation) rates [7, 10-11, 13-17], the coding for tests at 7 different rates, and the overall timescale of events in the context of real life time scenarios.

3.3 Ductile vs brittle behaviour Figure 5 shows work of fracture traces for 2 representative cases of fully ductile and semi-brittle fracture. The degree of ductility can be judged on the load/deformation traces and/or the fracture surface roughness $[7,12]$ as brittle fractures are associated with (i) precipitous drop in load and (ii) flat smooth fracture profiles. These two criteria could allow for classification of each failure as brittle or 
ductile. A ductility score between 1 (ductile) and 0 (brittle) was produced for each specimen based on these 2 criteria. The score values were added in 'bins' for age and strain rates and the percentage of samples that showed a ductile behaviour (\% ductile) was produce as a new variable.
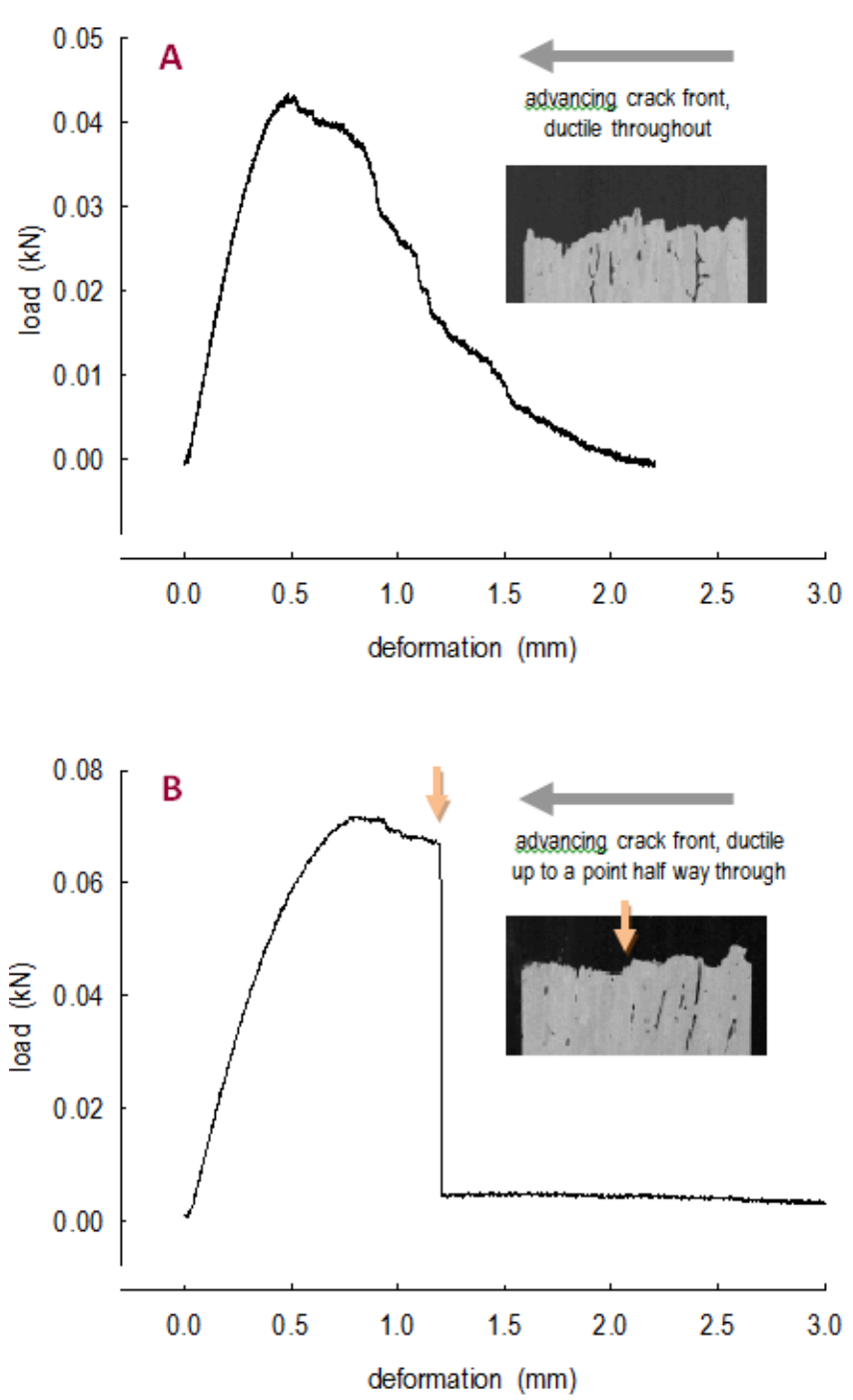

Figure 5. Load-deformation traces from two work of fracture (Wf) tests both for the 15 yrs old. (A) speed $5 \times 10-3 \mathrm{~mm} \mathrm{~s}-1$; (B) speed $5 \times 10-1 \mathrm{~mm} \mathrm{~s}-1$. Specimen in (A) broke in a ductile manner throughout the test and was given a 'ductility score' of 1. Specimen in (B) showed a ductile-to-brittle transition (DBT) half way through the test and was given a score of 0.5 . The small inset photos show the fracture profile along the advancing fracture front, which moves from the right to the left. Slow advancing fractures are generally ductile, appear rough and have relatively higher fractal dimension than fast moving fractures, which show smooth fracture surfaces.

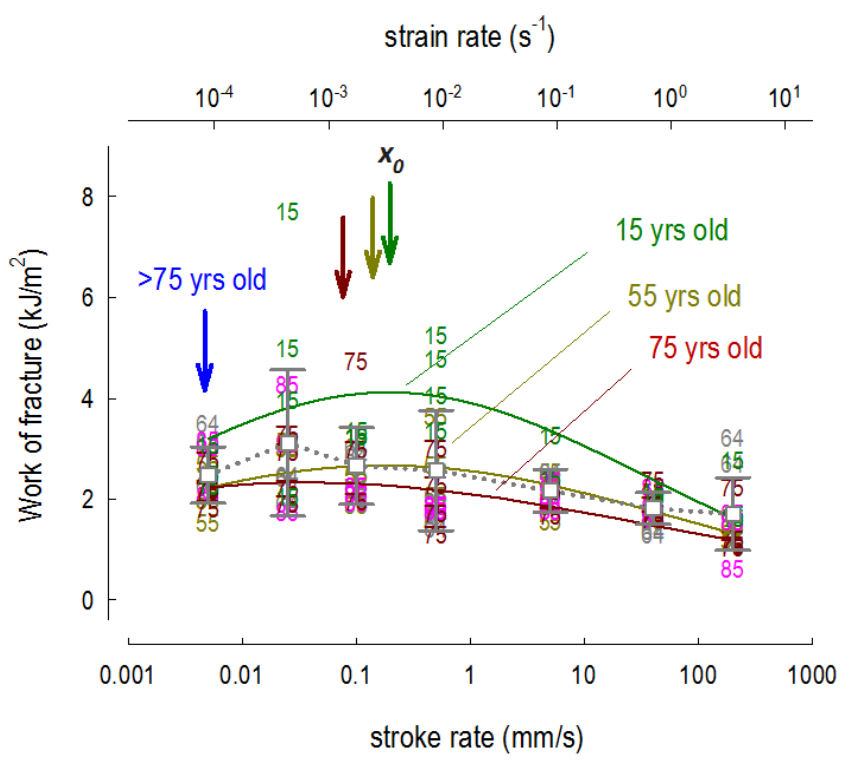

Figure 6. Work of fracture as function of strain rate, with age as symbols. Open squares are the means for Wf for each stroke rate across all 5 ages. The values show a peak from low stroke rates towards $0.01 \mathrm{~mm} / \mathrm{s}$ and then decline in accordance with other previous literature $[14,16-18,27]$. The bars are \pm one standard deviation off the mean. The smooth curves shown are nonlinear regressions of Lognormal, 3 parameter peak functions: $\mathrm{f}(\mathrm{x})=\mathrm{a}^{*} \exp [-0.5\{\ln (\mathrm{x} / \mathrm{x} 0) / \mathrm{b}\} 2]$, using dynamic fit routines in SigmaPlot v.10 (200 iterations, tolerance: $1 \times 10$ 10; full details and stats are given in e-Supplementary section); the $\mathrm{R} 2$ values of the fits ranged from $0.13-0.431$, and the $\mathrm{P}$ values from 0.0003 to 0.026 . For clarity only the lines for ages 55 , and two ages on either side of it, younger at 15 and older than 75 yrs are shown. The ductile-to-brittle transition, where the curves peak (arrows), decrease in value and shifts to lower strain rates with age; this can be seen by the $\mathrm{x} 0$ parameter values the peak of the Lognormal functions (full details in eSupplementary section).

3.4 Toughness vs strain rate Figure 6 shows the toughness of bone, $W_{f}$ versus strain rate and age. The data show a trend characteristic of other bone studies [14-16], which independently reported that bone exhibits a reduction in energy absorption with either age, or strain rate (for rates above $0.01 \mathrm{~s}^{-1}$ ). At low strain rates (below $10^{-3} \mathrm{~s}^{-1}$ ) $W_{f}$ increases with increasing strain rate, reaches a maximum at around $10^{-2} \mathrm{~s}^{-1}$ and then decreases beyond that 'threshold' rate. Such behaviour is characteristic of a DBT. Below the strain rates at peak $W_{f}$ it has been speculated [37] that thermally activated plasticity operates in the bone matrix. In this picture, as the strain rates increase, higher stresses are necessary to maintain plastic flow [37] and as this mechanism ceases to operate near 10-2 s- 1 , the $W_{f}$ curve levels off. 
In metals, a dynamic recovery by removal or rearrangement of defects facilitates plasticity. In the framework of the thermally-activated plasticity model of bone deformation [37] referenced above, at strain rates lower than about 10-2 s-1, recovery aids deformation by defect repair, but this help becomes less and less effective as strain rates approach this value. Is to be noticed that this threshold strain rate at maximum fracture work is close to the relaxation rate $r=1 / 75 \mathrm{~s}-$ $1=1.3310-2 \mathrm{~s}-1$ obtained from the timescales of strain redistribution after fracture in collagen and mineral crystals within bone [38,39]. In this situation defect repair and hardening balance each other. At higher strain rates recovery helps less and less to repeal any deformation damage, therefore, $W_{f}$ starts to decrease in value.

In the heterogeneous and anisotropic microstructure of bone even non-ductile pull out of fibres and lamellae produce surfaces that dissipate energy by friction in the process of separation [27]. Altogether the DBT in bone in not sharp and well defined, but its existence is beyond doubt $[18,27]$. It is remarkable, but understandable from the evolutionary biology point of view, that bone is toughest at strain rates when this is needed most; the physiological strain rates $[10,11]$ in walking and running are also about $3 \times 10^{-2} \mathrm{~s}^{-1}$. One observes a happy coincidence between physiologically occurring rates, spontaneous recovery rate $r$, and occurrence of maximum toughness. Fig.6 shows for the first time what the combined effect of these two factors is, and also that the peak in the $W_{f}$ curves has a tendency to shift with age to lower strain rate values. In other words, the maximum energy absorption reduces with age and at the same time the window of strain rate, at which bone achieves its optimum performance, shifts to slower rates.

\subsection{Ductile to Brittle Transition vs age Figure 7} shows the percentage of samples (in each combination of strain rate and age) exhibited ductile fracture (\%ductile) as a function of strain rate and age. Logistics sigmoidal curves were fitted to the data (esupplementary information). The DBT (at a likelihood of $50 \%$ between ductile or brittle) shows a shift between 15 and 85 years of age from a strain rate of $\sim 9 \times 10^{-3} \mathrm{~s}^{-1}$ to $\sim 7 \times 10^{-4} \mathrm{~s}^{-1}$, more than a factor of 10 difference. At lower specimen survival rates (i.e. at \%ductile $=10$, where only $10 \%$ of the samples managed to sustain a fully ductile fracture) the shift in strain rates is by a factor of 40 between old and young bone. The envelope between the extreme curves for old (85 yrs) and young (15yrs) bone shown in Fig.7 covers almost the entire range of speeds at which we load our femurs when walking, jumping or climbing stairs. More specifically it

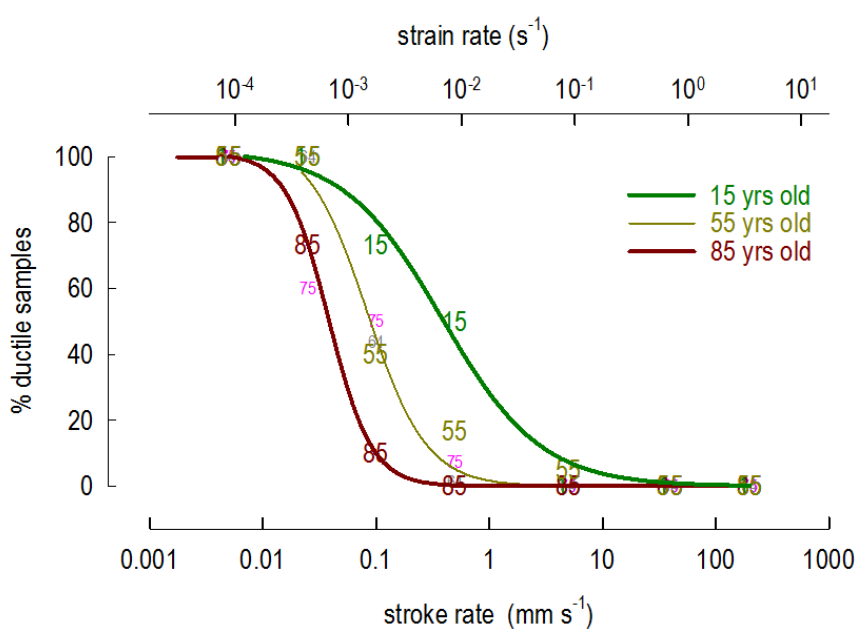

Figure 7. Percentage of ductile fractures versus strain rate and age. The smooth transition lines are logistics curves, nonlinear regressions of Sigmoidal, 3 Parameter functions: $f(x)=a /\{1+\exp (-(x-x 0) / b)\}$, using dynamic fit routines in SigmaPlot v.10 (1000 iterations, tolerance: $1 \times 10 \quad 10$; full details and stats are given in e-Supplementary section); the R2 values of the fits ranged from $0.96-1.00$, and the $P$ values $<0.001$ for all fits. The transition curves show a shift with $\mathrm{x}$, the strain rate, which varies as a factor of 10 and 40 between the youngest and oldest individual. In other words, an everyday loading event, at a moderate strain rate, which for a young person would go unnoticed, would have detrimental effects in the older person resulting in a brittle fracture $(100 \%$ brittle for any strain rate above $0.01 \mathrm{~s}-1$ ).

also shows, for instance, that while young bone is ductile at jumping speeds, old bone is brittle. Moreover, as shown earlier in Fig. 6 the toughness (as energy absorption) that can be sustained at these rates decreases with age.

We can, therefore, for the first time show that bone is most fracture resistant at strain rates such as needed for everyday living and survival - its toughness peaks between $10^{-4}$ and $10^{-1} \mathrm{~s}^{-1}$. Secondly, with age the peak strength decreases, and the peak shifts to lower speeds - the elderly being both weak and slow. Thirdly, the signature of the strain curve varies with deformation speed. At low rates the elastic line is followed by a few percent plasticity, at high rates the fracture is catastrophic and occurs at the elastic limit. Fourthly, optical inspection confirmed the classification of fractures as ductile or brittle: ductility is associated with rough plastically deformed fracture surface and fibre pull-out, brittleness with breakage of fibres along a cleavage plane.

3.6 Biophysical nature of events As mentioned earlier, by using X-ray diffraction in a synchrotron 
radiation source [38,39] we have observed that after being stretched to failure, mineral crystals and collagen in bone had different relaxation rates. Whereas mineral crystals relaxed immediately, the rate of recoil in collagen was $r=1 / 75 \mathrm{~s}-1=1.3310-2 \mathrm{~s}-1$. While unloading bone samples before failure had identical relaxation rates. Thus, this was interpreted as the typical timescale of self-healing of bone under natural conditions, being induced by the load transfer between mineral crystals and collagen by the interfibrillar matrix. This interpretation was favoured, as previous investigations using synchrotron radiation showed the importance of the interfibrillar matrix in the mechanical deformation of bone tissue [40] and were then intensively investigated, as shown in a review article [41]. Nevertheless, one cannot rule out that also sliding mechanisms in the triple helix molecules play a role. Such sliding in collagen was observed and quantified in early pioneering works using in-situ X-ray scattering from high brilliance synchrotron radiation sources [42]. Both effects, sacrificial bonds in the interfibrillar matrix $[43,44]$ or collagen sliding induced viscosity [42] and collagen cross-links [45] could contribute to plasticity in bone. The observation in Figure 6 is that at low strain rates, below the recovery rate $\mathrm{d}$ (2) $\mathrm{dt}<\mathrm{r}$, the work of fracture is lowers, but the same is observed for very high strain rates. The mechanisms that are at play in these two extremes (very low strain rates and very high rates) are however very different. Very low strain rates involve creep rupture phenomena and accumulation of damage with loading over prolonged times. At high strain rates the contribution to the mechanics by the organic matrix component may be what makes all the difference. We see here that the maximum in the work of fracture shifts to lower strain rates with increasing age. In the scientific literature on metal behaviour this would correspond to a balance of recovery and hardening, where recovery is less effective with age. For bone, one may speculate about the underlying mechanisms: A possible interpretation could be that with strain rate the relative contribution of sliding mechanism in collagen molecules vs recovery offered by sacrificial bonds shifts. Quite possibly at higher strain rates, time is not sufficient for the organic matrix based self-healing mechanisms of bone to confer toughness. Recovery of sacrificial bonds is - as a molecular mechanism - probably age independent, whereas the sliding viscosity reduces with age probably due to increasing cross-links of collagen. Depalle et al. [46] that the enzymatic crosslinking profile is very different in $15 \mathrm{yr}$ olds vs $75 \mathrm{yr}$ olds. The broad view is that the organic matrix changes with age [45] and that interplays with fracture outcome during ageing [47] and as shown when comparing age/sex matched donors with and without fractures where the organic matrix quality was evidently different [48]. However, other work examining both enzymatic and non-enzymatic cross links showed inconclusive results as far as toughness is concerned in paired anatomical locations [49] . As for ageing effects these have not only been observed in bone [50], but also in ligaments or cartilage $[51,52,53]$. Taking these observations together, the question is still open, whether structural changes in the organic matrix, probably alongside changes in the inorganic matrix and the well known documented changes in bone density, mineral content and porosity, are the most important parameter for deterioration of bone's quality with age.

\section{Conclusions}

It is well accepted that bone is a semi-brittle material with an ability to exhibit plasticity and ductility when the circumstances allow it, whereby either the loading environment (applied strain rates) or the ambient conditions (humidity/dryness) define the degree of its ductility. However, the intrinsic ability of bone to exhibit ductility either by natural design, or in ontogeny (ageing), or in disease has not been the result of bespoke attention in the past. More particularly there are no studies, which examine the former (conditions) in conjunction with the latter (ageing/disease); the extrinsic in relation to the intrinsic phenomena. Here for the first time we have shown that toughness of human femoral bone (energy absorbed per unit fracture area) reduces with age across a wide spectrum of strain rates, for slow and fast loading events. Bone also exhibits a change from ductility to brittleness as loading rates increase. Its toughness shows a maximum at the physiologically occurring strain rates of $10^{-3} \mathrm{~s}^{-1}$ to $10^{-2} \mathrm{~s}^{-}$ 1 , which may be a result of some optimisation and natural design routines. But more importantly, the peak of this performance shifts towards lower values of strain rate with age. Use of logistics curves to express the relative degree of plasticity and brittleness versus strain rate showed that there is also a shift of these DBT curves with ageing towards lower strain rates by a factor of 10 to 40 by the age of $85 y$ r old on either side of skeletal maturity. The combined effect of a reduced absolute toughness value, and a DBT shift to lower strain rates, produce a new powerful paradigm, which allows us to demonstrate mechanically, and uniquely, how human bone becomes increasingly brittle with age.

\section{Acknowledgments}

PZ acknowledges the support provided by EPSRC (UK) under the 'Point-of-Care High Accuracy Fracture Risk Prediction' EP/K020196/1 grant. HP acknowledge the support from the 
Austrian Science Funds (FWF), proj. nr. I449 and the German Research Foundation (DFG), proj. nr. PE 1732/1-2, which allowed testing in the synchrotron source in Trieste, Italy. The authors are thankful to Ms V.Remy an Erasmus exchange student for help with the mechanical testing; Ms V.Wise for the work on chemical composition and bone analysis data; and $\mathrm{Dr}$ E.Minnock for building the kinematics computer model to demonstrate slips, falls and accidents of Fig.1. The tests were carried out in the biomechanics labs of Cranfield University, UK. The authors thank those bereaved families who kindly donated tissues to be used for the benefit of others. PZ acknowledges the support provided by the UK Department of Transport under the BOSCOS (Bone Scanning for Occupant Safety) project for which ethical consent (BOSCOSMr.C.Curwen CI REC ref. 01/179G) was obtained in conjunction with the Gloucester and Cheltenham NHS Trust hospitals.

\section{Authors' contributions}

$\mathrm{PZ}$ was involved throughout the testing and running of the project. HOKK, HP contributed in the conception and writing up of the article as well as running complementary tests in the synchrotron source that underpinned the work presented here. All authors discussed the results and contributed to the final manuscript.

\section{Competing financial interests}

The authors declare that they have no competing interests.

\section{Data accessibility}

Data for this ms upon acceptance will be available through the Cranfield University CORD data depository and preservation system at https://cranfield.figshare.com

\section{References}

1 Cooper, C. The epidemiology of fragility fractures: Is there a role for bone quality? Calcified Tissue Intern. 53 (1993) S23S26.

$2 \quad$ Gillet, P. \& Reginster, J.Y. Increased number of hip fractures. Lancet 353 (1999) 2160-66.

3 Zioupos, P. \& Currey, J.D. Changes in the stiffness, strength, and toughness of human cortical bone with age. Bone 22 (1998) 57-66.

4 Dicken, A.J., Evans, J.P.O., Rogers, K.D., Stone, N., Greenwood, C., Godber, S.X., Clement, J.G., Lyburn, I.D., Martin, R.M., Zioupos, P. Classification of fracture and non-fracture groups by analysis of coherent X-ray scatter. Scientific Reports 6 (2016) 29011, doi: 10.1038/srep29011.

5 Ma, S., Goh, E.L., Jin, A., Bhattacharya, R., Boughton, O.R., Patel, B., Karunaratne, A., Vo, N.T., Atwood, R., Cobb, J.P., Hansen, U., Abel, R.L. Long-term effects of bisphosphonate therapy: Perforations, microcracks and mechanical properties. Scientific Reports 7 (2017) 43399, doi: 10.1038/srep43399.

6 Zioupos, P. Ageing human bone: factors affecting its biomechanical properties and the role of collagen. J. Biomaterials Appl. 15 (2001) 187-229.

7 Currey, J.D., Brear, K., Zioupos, P. The effects of ageing and changes in mineral content in degrading the toughness of human femora. J Biomechanics 29 (1996) 257-60.

8 Fradet, L, Cliche, F, Petit, Y, Mac-Thiong, JM, Arnoux, PJ. Strain rate dependent behavior of the porcine spinal cord under transverse dynamic compression. Proc IMech - H -J Engng Medicine 230 (2016) 858-66.

9 Enns-Bray, W.S, Ferguson, S.J; . Strain rate dependency of bovine trabecular bone under impact loading at sideways fall velocity. J Biomechanics 75 (2018) 46-52.

10 Burr, D.B., Milgrom, C., Fyhrie, D., et al., In vivo measurement of human tibial strains during vigorous activity. Bone 18 (1996) 405-10.

11 Currey, J.D. Bones: structure and mechanics. (Princeton University Press, Princeton, 2002).

12 Zioupos, P., Kaffy, C., Currey. J.D. Tissue heterogeneity, composite architecture and fractal dimension effects in the fracture of ageing human bone. Intern. J. Fracture 139 (2006) 407-24.

13 Moran. S.G., McGwin, G., Metzger, J.S., et al. Relationship between age and lower extremity fractures in frontal motor vehicle collisions. J. Trauma-Injury Infection \& Critical Care 54 (2003) 261-65.

14 McElhaney, J.H. Dynamic response of bone and muscle tissue. J. Appl. Physiology 21, (1966) 1231-36.

15 Robertson, D.M. \& Smith, D.C. Compressive strength of mandibular bone as a function of microstructure and strain rate. J. Biomechanics 11 (1978) 455-71.

16 Crowninshield, R.D. \& Pope, M.H. The response of compact bone in tension at various strain rates. Annals Biomed. Engineering 2 (1974) 217-25.

17 Evans, G.P., Behiri, J.C., Vaughan, L.C., et al. The response of equine cortical bone to loading at strain rates experienced invivo by the galloping horse. Equine Veterinary J. 24 (1992) 125-28.

18 Kirchner, H. Ductility and brittleness of bone. Intern. J. Fracture 139 (2006) 509-17.

19 Hansen, U., Zioupos, P., Simpson, R., et al. The Effect of Strain Rate on the Mechanical Properties of Human Cortical Bone. J. Biomechanical Engineering/Trans ASME 130 (2008) 011011-1-8.

20 Ural A, Zioupos P, Buchanan D, Vashishth D. The effect of strain rate on fracture toughness of human cortical bone: a finite element study. J Mech Behav Biomed Mater. 4 (2011) 1021-32. 
Ural A, Zioupos P, Buchanan D, Vashishth D. Evaluation of the influence of strain rate on Colles' fracture load. $J$ Biomechanics 45 (2012) 1854-57.

Asgharpour Z, Zioupos P, Graw M, Peldschus S. Development of a Strain Rate Dependent Material Model of Human Cortical Bone for Computer-aided Reconstruction of Injury Mechanisms. For Science Intern, 236 (2014) 109-16. cortical bone toughness: A comparative study of four paired anatomical sites. J Mech Behav Biomed Materials 71 (2017) 223-30.

Zioupos, P., Hansen, U., Currey, J.D. Microcracking damage and the fracture process in relation to strain rate in human cortical bone tensile failure. J Biomechanics 41 (2008) 2932-39.

Rho, JY., Kuhn-Spearing, L., Zioupos, P., Mechanical properties and the hierarchical structure of bone. Medical Engineering Physics 20 (1998) 92-102.

Zioupos, P. Recent developments in the study of failure of solid biomaterials and bone: 'fracture' and 'pre-fracture' toughness. Mater. Sci. Engineering C 6 (1998) 33-40.

Tattersall, H.G. \& Tappin, G. The work of fracture and its measurement in metals, ceramics and other materials. J. Mater. Science 1 (1966) 296-301.

29 Alman, B. and Frasca, P. Fracture failure mechanisms in patients with osteogenesis imperfecta. Journal of orthopaedic Research 5 (1987) 139-43.

Brear, K., Currey, J.D., Pond, C.M. and Ramsay, M.A. The mechanical properties of the dentin and cement of the tusk of the narwhal Monodon monoceros compared with those of other mineralised tissues. Archives of oral Biology 35 (1990) 61521.

Moyle, D.D. and Bowden, R.W. Fracture of human femoral bone. Journal of Biomechanics 17 (1984) 203-13.

\section{dental Research 63 (1984) 1362-68.}

Rogers, L.L. and Moyle, D.D. Effect of specimen size on work-of-fracture measurements. Journal of Biomechanics 21 (1988) 919-26.

ASTM C1421-01b. Standard Test Methods for Determination of Fracture Toughness of Advanced Ceramics at Ambient Temperature, 2007.

Anderson, T.L. Fracture Mechanics, Fundamentals and Applications. (2nd Ed., CRC Press, Boca Raton, FL, 1995).

Gere, J.M., Timoshenko, S.P. Mechanics of Materials, (2nd Ed., PWS Engineering, Boston MA, 1984).

Gupta, H.S., Fratzl, P., Kerschnitzki, M., et al. Evidence for an elementary process in bone plasticity with an activation enthalpy of 1 eV. J. Royal Soc. Interface 4 (2007) 277-82.

38 Puchegger, S., Rennhofer, H., Loidl, D., et al. Relaxation of human bone at the nanoscale. (Austrian SAXS Beamline at Elettra: Annual Report 2005, pp.98-100, 2005).

39 Akbarzadeh J, Puchegger S, Stojanovic A, Kirchner H.O.K., Binder W.H., Bernstorff S, Zioupos P, Peterlik H. 'Timescales of self-healing in human bone tissue and polymeric ionic liquids.' Bioinspired, Biomimetic and Nanobiomaterials 3 (2014) 123-30.

40 Gupta HS, Wagermaier W, Zickler GA, Raz-Ben Aroush D, Funari SS, Roschger P, Wagner HD, Fratzl P. Nanoscale Deformation Mechanisms in Bone, Nano Lett. 5 (2005) 2108-2111. doi.org/10.1021/nl051584bH

41 Stock SR, The Mineral-Collagen Interface in Bone, Calcif Tissue Int. 97 (2015) 262-280. doi: 10.1007/s00223-015-9984-6

42 Mosler E, Folkhard W, Knörzer E, Nemetschek-Gansler H, Nemetschek Th, Koch MHJ. Localization of stress-induced molecular rearrangements in collagen. Colloid and Polymer Science, 263 (1985) 87-88.

43 Fantner, G.E., Hassenkam, T., Kindt, J.H., et al. Sacrificial bonds and hidden length dissipate energy as mineralized fibrils separate during bone fracture. Nature Materials 4 (2005) 612-16.

44 Fantner, G.E., Oroudjev, E., Schitter, G., et al. Sacrificial Bonds and Hidden Length: Unraveling Molecular Mesostructures in Tough Materials. Biophysical J. 90 (2006) 1411-18.

45 Unal M, Creecy A, Nyman JS. The role of matrix composition in the mechanical behaviour of bone. Curr Osteopor. Rep. 16 (2018) 205-215.

46 Depalle B, Duarte AG, Fiedler IAK, Pujo-Menjouet L, Buehler MJ, Berteau J-P. The different distribution of enzymatic collagen cross-links found in adult and children bone result in different mechanical behaviour of collagen. Bone 110 ( 2018) 107-114.

47 Zioupos, P., Currey, J.D., Hamer, A.J. The role of collagen in the declining mechanical properties of aging human cortical bone. J. Biomed. Mater. Research 45 (1999) 108-16.

Rokidi S, Paschalis EP, Klaushofer K, Vennin S, Desyatova A, Turner JA, Watson P, Lappe J, Akhter MP, Recker RR. Organic matrix quality discriminates between age- and BMD-matched fracturing versus non-fracturing post-menopausal women: A pilot study. Bone, 127 (2019) 207-214, doi.org/10.1016/j.bone.2019.06.017 toughness and collagen cross-links on paired anatomical locations. Bone 112 (2018) 201-211. 
Quiroga, JMP, Wilson, W, Ito, K, van Donkelaar, CC. (van Donkelaar, C. C. Relative contribution of articular cartilage's constitutive components to load support depending on strain rate. Biomech Modelling Mechanobiology 16 (2017) $151-58$.

51 Quiroga, JMP, Wilson, W, Ito, K, van Donkelaar, CC. The effect of loading rate on the development of early damage in articular cartilage. Biomech Modelling Mechanobiology 16 (2017) 263-73.

52 Henao-Murillo, L, Ito, K, van Donkelaar, CC. Collagen Damage Location in Articular Cartilage Differs if Damage is Caused by Excessive Loading Magnitude or Rate. Annals Biomed Engineering 46 (2018) 605-15.

53 Karunaratne, A, Li, SM, Bull, AMJ. Nano-scale mechanisms explain the stiffening and strengthening of ligament tissue with increasing strain rate, Scientific Reports 8 (2018) 3707, doi: 10.1038/s41598-018-21786-z. 